\title{
Parameter Estimation of Three-Phase Induction Motor by Using Genetic Algorithm
}

\author{
Seesak Jangjit ${ }^{\dagger}$ and Panthep Laohachai*
}

\begin{abstract}
This paper suggests the techniques in determining the values of the steady-state equivalent circuit parameters of a three-phase induction machine using genetic algorithm. The parameter estimation procedure is based on the steady-state phase current versus slip and input power versus slip characteristics. The propose estimation algorithm is of non-linear kind based on selection in genetic algorithm. The machine parameters are obtained as the solution of a minimization of objective function by genetic algorithm. Simulation shows good performance of the propose procedures.
\end{abstract}

Keywords: parameter estimation, induction motor, genetic algorithm

\section{Introduction}

In ac induction motor drives, the electrical parameters are, generally, determined via the classical analysis no-load and locked-rotor test may be obtained from actual machine test or from data supplied by the manufacturer [1]. Estimation of the performance, done by plotting the steady-state slip curve. Generally to obtain the parameter, one must use the equivalent circuit relations and experimental results obtained from the above-mentioned classical analysis. Therefore, the parameter values obtained by direct classical approaches or experimental can reveal significant difference in the entire range of slip varying from 0 to 1 . To describe the performance of the induction machine more precisely and to reduce the differences between the estimated and real performance, one must modify the parameters obtained from the classical analysis [2]. To achieve this purpose in motor, the use of identification algorithms base on the artificial algorithm appears to be promising approach.

In this study it is ignored that the effect of measurement errors, disturbances, random signals, and core losses on the estimated parameters. Since the equation relating the phase current to slip and the circuit parameters involve many variables and are nonlinear, parameters can have difference values in case of the change of load. This fact does not enable to one directly use the many parameter estimation procedures existing in the literature [3]-[6].

The estimation method described in this paper differs from other approaches in this following ways. Ansuj and Shokooh [1] presented a parameter estimation procedure that required knowledge of full load power factor and efficiency. Other method of parameter estimation employ least

$\dagger \quad$ Corresponding Author : Dept. of Electrical and Computer Engineering, Kasetsart University, Sakon Nakhon ,Thailand.

(fsessj@csc.ku.ac.th)

* Dept. of Electrical Engineering, Kasetsart University, Bangkok, Thailand .(fengptl@ku.ac.th)

Received 15 February 2008; Accepted 9 July 2009 square analysis of data generated during actual transient conditions.

In this paper, obtaining optimal parameter of the equivalent circuit of three-phase induction machine is suggested by genetic algorithm. During the execution of the estimation algorithm, we use the three points of steadystate data of both the input power the stator current.

\section{Induction Motor Model}

A three-phase induction machine supplied with a threephase symmetrical voltage source can be described using the equivalent circuit shown in Fig. 1.

A listing of the parameters in this equivalent circuit and their dependencies on the machine speed as treated in this paper follows. All circuit parameters are in ohm referred to stator winding.

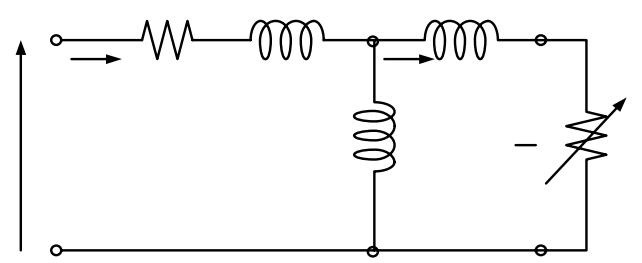

Fig. 1. Equivalent circuit representing steady-state of polyphase induction motor

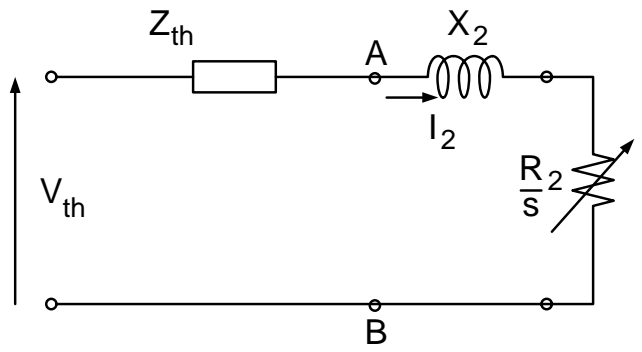

Fig. 2. Equivalent circuit simplified by Thevenin's theorem 
$R_{1}$ stator winding resistance

$X_{1}$ stator leakage reactance

$R_{2}$ rotor winding resistance referred to stator side

$X_{2}$ rotor leakage reactance referred to stator side

$X_{m}$ magnetizing reactance referred to stator side

$S \quad$ slip speed

$V_{1}$ terminal voltage

$I_{1}$ stator current

$I_{2}$ rotor current referred to stator side

In case the stator current and the input power, the equation for induction motor can be determined from the circuit of Fig. 2 and are expressed as following:

$$
\begin{gathered}
V_{t h}=\frac{V_{1}\left(j X_{m}\right)}{R_{1}+j\left(X_{1}+X_{m}\right)} \\
Z_{t h}=\frac{j X_{m}\left(R_{1}+j X_{1}\right)}{R_{1}+j\left(X_{1}+X_{m}\right)}
\end{gathered}
$$

From Fig. 2, the rotor current can be determined by the following equation.

$$
I_{2}=\frac{V_{t h}}{Z_{t h}+j X_{2}+\frac{R_{2}}{S}}
$$

For determined the stator current we can reduce the part of rotor equivalent circuit as the below.

Where $Z_{e q}$ determined from the following procedures.

$$
\begin{aligned}
A & =\frac{1}{j X_{m}}+\frac{1}{\left(R_{2} / s+j X_{2}\right)} \\
Z_{e q} & =\frac{1}{A} \\
I_{1} & =\frac{V_{1}}{\left(R_{1}+j X_{1}\right)+Z_{e q}} \\
I_{1} & =\left|I_{1}\right| \angle \theta \\
p f & =\cos \theta \\
P_{i n} & =3\left|V_{1}\right|\left|I_{1}\right| p f
\end{aligned}
$$

\section{Estimation Framework}

In order to determine model parameters for the slip curve of equivalent circuit, reference [2] uses the nonlinear curve fitting problem stated as the solution of the following minimization problem:

$$
\min _{\Phi \in \Omega} J(\Phi)=\frac{1}{N} \sum_{i=1}^{N}\left[y_{i}-y\left(s_{i}, \Phi\right)\right]
$$

where $J(\Phi)$ is objective function obtained by the sum of the square of the differences between the experimental and calculated slip curve, $\Omega$ is the parameter space depending on the number of parameters to estimated, $y_{i}$ is the experimental data value collected from machine, $y\left(s_{i}, \Phi\right)$ is non-linear function relating the measurement data, the circuit parameters, and the slip, and $\Phi$ is parame- ter vector pertaining to $\Omega$. Therefore, in case of dimension of parameter vector $\Phi$ is defined as:

$$
\Phi=\left[\begin{array}{lllll}
R_{1} & R_{2} & X_{1} & X_{2} & X_{m}
\end{array}\right]
$$

The above mention specific equation is for depend on the kind of available experimental data and for obtaining a parameter vector that minimize the quadratic performance index defined by (10). In this case, since one must deal with a non-linear algorithm to acquire the desired solution, some numerical problems may arise or direct approach would require writing down the normal equations for the solving them. The method for numerical minimization of performance index (10) might be modified to update the estimated parameter vector according load change.

\section{Genetic Algorithm}

\subsection{Fundamental of Generic Algorithm}

This optimization method described in [7] and [8] by George K. Stefopoulos, et al. in the following.

Genetic Algorithms are optimization methods inspired by natural genetics and biological evolution. They manipulate strings of data, each of which represents a possible problem solution. These strings can be binary strings, floating-point string, or integer strings, depending on the way the problem parameters are code into chromosomes. The strength of each chromosome is measured using fitness values, which depend only on the value of the problem objective function for the possible solution represented by the chromosome. The stronger strings are retained in the population and recombined with other strong strings to produce offspring. Weaker ones are gradually discarded from the population. The processing of strings and the evolution of the population of candidate solutions are performed based on probabilistic rules. Reference [7] provides a comprehensive description of genetic algorithms.

\subsection{Chromosome Representation}

Two types of representations have been investigated, binary and real.

\subsection{Creation of Initial Population}

The initial population of candidate solution is created randomly.

\subsection{Evaluation of Candidate Solutions}

Each candidate solution represents a parameter vector $\Phi$; the evaluation of each candidate solution is based on the objective function value $J(\Phi)$. Note that the objective function value is obtained after system simulation.

The purpose of the process is to solve a minimization problem; the objective function to be minimized is defined as 


$$
F(\Phi)=J(\Phi)+K
$$

where $K$ is small positive real number used as scaling coefficient, in order to avoid problems that may arise as $J(\Phi)$ approaches zero, and to control problem like premature convergence.

\subsection{Reproduction}

Reproduction refers to the process of selecting the best individuals of the population and copying them into a "mating pool". These individuals from an intermediate population. Three types of reproduction process are implemented in this work:

1) Roulette-wheel selection,

2) Tournament selection with user-defined window,

3) Deterministic sampling based on the fitness proportionate selection scheme.

\subsection{Crossover Operation}

In binary representation the following four types of crossover are used:

1) 1-point crossover

2) 2-point crossover

3) Uniform crossover, which is a crossover operator that swaps only single bits between the two parent binary strings.

4) Multi-point crossover, in which one crossover point is selected, randomly, for each parameter represented in the chromosome, and thereafter, 1-point crossover is performed in each parameter.

In floating-point representation the crossover types used are:

1) 1-point crossover,

2) 2-point crossover,

3) Uniform crossover,

4) Arithmetical crossover.

\subsection{Mutation Operation}

When binary coding is used, the genetic algorithm mutation simply changes a bit from " 0 " to " 1 " or vice versa. The bits that undergo mutation are chosen based on a probability test. The probability of mutation is generally set to a small value, about 0.001 to 0.01 .

In real representation, two mutation operators are implemented: uniform and non-uniform mutation.

1) Uniform mutation: This operator is analogous to the binary operator, but it applies to real values instead of binary bits; it randomly replaces the parameter value with another one from the appropriate interval:

2) Non-Uniform mutation: This mutation type is described in [8] and it is responsible for the fine-tuning capabilities of the real-codes GA. If a parameter $k$ of value $u_{k}$ of a candidate solution is selected for mutation, its value is changed to $u_{k}^{\prime}$;

$$
u_{k}^{\prime}=\left\{\begin{array}{l}
u_{k}+\Delta t, U B-u_{k} \\
u_{k}-\Delta t, u_{k}-L B
\end{array}\right.
$$

where $u_{k}^{\prime}$ depending on whether a random binary digit is 0 or 1 . $L B$ and $U B$ are the lower and upper bounds of the individual parameter $k$ belong to. The function $\Delta(t, y)$ return a value in the range $[0, y]$ such that the probability of $\Delta(t, y)$ being close to 0 increases as the current generation number, $t$, increases. This property causes this operator to uniformly search the space at initial stages, when $t$ is small, and very locally at later stages. The function used is

$$
\Delta(t, y)=y \cdot\left(1-\gamma^{\left(1-\frac{t}{T}\right) b}\right)
$$

where $\gamma$ is a random number in [0,1], $T$ is the maximal generation number, and $b$ is a parameter determining the degree of non-uniformity [8].

In real representation, since parameters do not change during crossover, but are just recombined differently (except for the arithmetical crossover), the only way of affecting their values is by the mutation operator. Moreover, the mutation probabilities used are greater than the ones in binary representation and may reach up to 5\% [7].

\subsection{Creation of the Next Generation}

After mutation is completed, the children population is created and the previous population is replaced by the new generation. Children are evaluated and the fitness function for each individual is calculated. The procedure is repeated until the termination criterion is met, defined by a maximum number of generation.

\section{Simulation}

The illustrate the application of the propose method a 3 hp, $380 \mathrm{~V}, 50 \mathrm{~Hz}$ three-phase induction motor was selected. The performance of machine between $0-1$ of slip difficult to determine in practice. Therefore, a computer program was developed for PC to calculate the steady-state performance from the real parameter. The input data of the propose method are the stator current, the input power, and the power factor, that corresponded with the $0-0.1$ of slip.

The genetic algorithm suggested in this paper is simulated and compared with true values. Objective function $J_{1}(\Phi)$ is used but object function $J_{2}(\Phi)$ is introduced for more optimal parameter selection as follow:

$$
\begin{aligned}
J_{1}(\Phi)= & \frac{1}{N} \sum_{i=1}^{N}\left[I_{t}\left(s_{i}\right)-I_{c}\left(s_{i}, \Phi\right)\right]^{2} \\
& +\frac{1}{N} \sum_{i=1}^{N}\left[P_{i n_{i}}\left(s_{i}\right)-P_{i n_{c}}\left(s_{i}, \Phi\right)\right]^{2}
\end{aligned}
$$




$$
\begin{aligned}
J_{2}(\Phi)= & \frac{1}{N} \sum_{i=1}^{N}\left[I_{t}\left(s_{i}\right)-I_{c}\left(s_{i}, \Phi\right)\right]^{2} \\
& +\frac{1}{N} \sum_{i=1}^{N}\left[P_{i n_{i}}\left(s_{i}\right)-P_{i n_{c}}\left(s_{i}, \Phi\right)\right]^{2} \\
& +\frac{1}{N} \sum_{i=1}^{N}\left[p f_{t}\left(s_{i}\right)-p f_{c}\left(s_{i}, \Phi\right)\right]^{2}
\end{aligned}
$$

where $I, P_{i n}$, and $p f$ are the amplitude of stator current, power input, and power factor, respectively. Also, $t$ and $c$ are the quantity of simulated by true value and genetic algorithm selection, respectively.

\section{Conclusion}

This paper investigates the application of genetic algorithm for the estimation of steady-state models of induction motor. The main advantages of the proposed methodology are using only 3-5 of input data points required, it flexibility, the simplicity of its mechanism, and the good result even for bad initial of parameters.

The propose method has been successfully applied to the dynamic estimation of the other machine models. The obtained results demonstrate the feasibility and practicality of the proposed genetic algorithm approach.

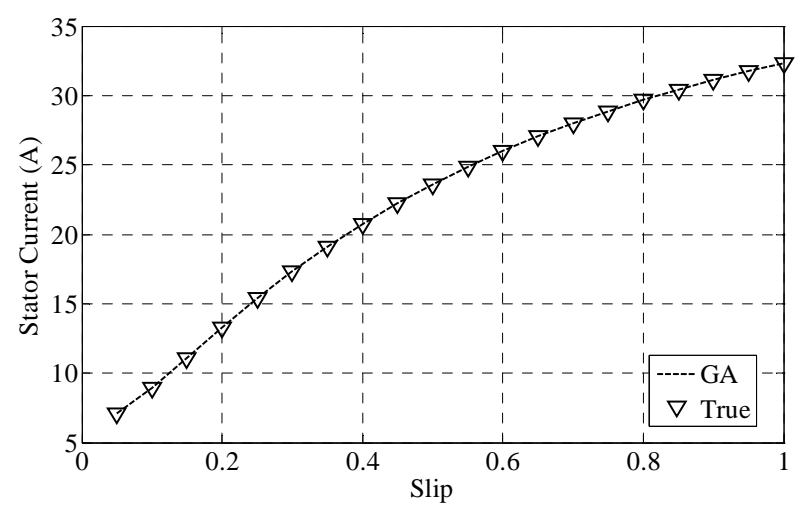

Fig. 3. Variation of $I_{1}(s)$ by genetic algorithm selection and true values

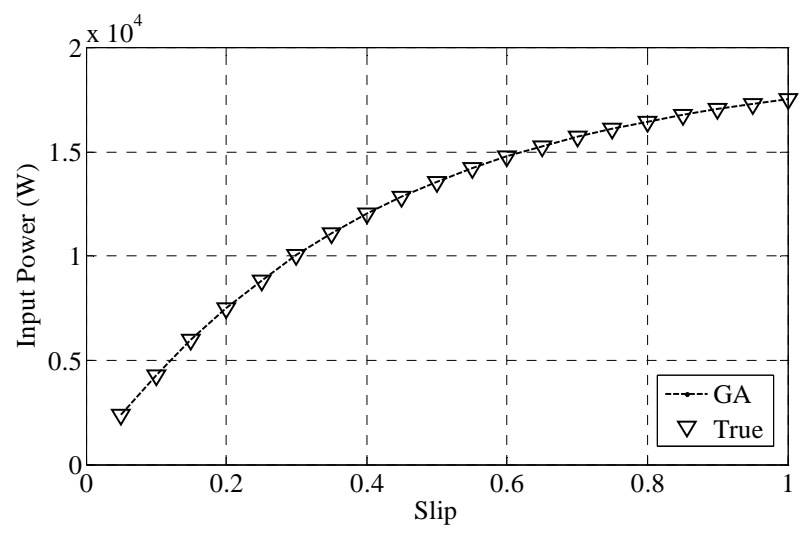

Fig. 4. Variation of $P_{i n}(s)$ by genetic algorithm selection and true values

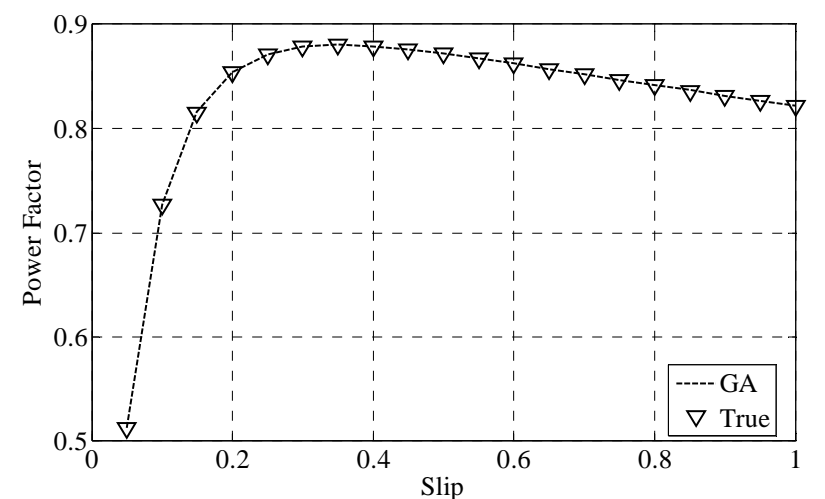

Fig. 5. Variation of $p f(s)$ by genetic algorithm selection and true values

Table 1. The caption must be followed by the table

\begin{tabular}{c|c|c|c|c|c}
\hline gen & $R_{1}$ & $R_{2}$ & $X_{1}$ & $X_{2}$ & $X_{m}$ \\
\hline True & 2.933 & 3.058 & 1.555 & 2.225 & 32.235 \\
\hline Initial & 1 & 1 & 1 & 1 & 1 \\
\hline$J_{1}(\Phi)$ & 2.933 & 2.966 & 2.041 & 1.680 & 31.749 \\
\hline$J_{2}(\Phi)$ & 2.933 & 3.070 & 1.490 & 2.299 & 32.302 \\
\hline
\end{tabular}

\section{Acknowledgements}

This work was supported by Kasetsart University Chalermphrakiat Sakon Nakhon Province Campus.

\section{References}

[1] S. Ansuj and, F. Shokooh, and R. Schinzingger, "Parameter Estimation for Induction Machines Based on Sensitivity Analysis," IEEE Transl, on industry application, .vol. 25, no 6, pp. 1035-1040.

[2] Dong Hwa Kim, and Jea Hoon Cho, "Parameter Estimation of a squirrel-Double Cage Induction Motor Using Clonal Selection of Immune Algorithm,” IEEE Industrial Electronics Society, Busan, Korea, p. 11901194.

[3] P.Vaclavek and P. Blaha, "Lypunov-Function-Based Flux and Speed Observer for AC Induction Motor Sensorless Control and Parameters Estimation,” IEEE Transl, on industry Electronics, .vol. 53, no 1, pp. 138-145.

[4] M. Calvo and O.P. Malik, "Synchronous Machine Steady-State Parameter Estimation Using Neural Networks,” IEEE Transl, on Energy Conversion, .vol. 19, no 2, pp. 237-244.

[5] D. J. Atkinson, P. P. Acarnley, and J.W. Finch, “Observers for Induction Motor State and Parameter Estimation,” IEEE Transl, on industry Applications, vol. 27, no 6, pp. 1119-1127.

[6] K. Wang, J Chiasson, M. Bodson, and L.M. Tolbert, 
“A Nonlinear Least-Squares Approach for Identification of the Induction Motor Parameters," Proc. 43th IEEE Conference on Decision and Control, December 14-17, 2004, Atlantis, Paradise Island, Bahamas, pp. 3856-3861.

[7] G. K. Stefopoulos, P.S. Georgilakis, N.D.Hatziargyriou, and A .P. Sakis Meliopoulos, "A Genetic Algorithm Solution to the Governor-Turbine Dynamic Model Identification in Multi-Machine Power System,” Proc. $44^{\text {th }}$ IEEE Conference on Decision and Control, and the European Control Conference 2005, December 12-15, 2005, pp. 1288-1294.

[8] Z.Michalewicz, Genetic Algorithms + Data Structures = Evolution Programs. New York: SpringerVerlag, 1996.

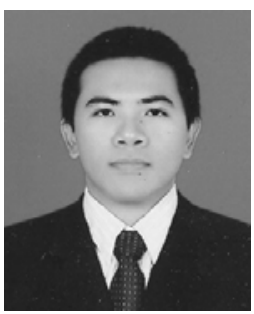

Seesak Jangjit He received B.Eng and M.Eng degrees in electrical engineering from Kasetsart University, Thailand. Currently, he is lecturer at Kasetsart University, Sakon Nakhon Campus. His research interests are electrical machines, power system dynamic and control, and power system satiability.

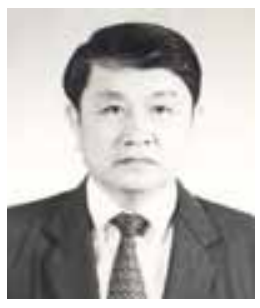

Panthep Laohachai He received B.Eng and M.Eng degrees in electrical engineering from Chulalongkorn University, Bangkok, Thailand and Ph.D. in electrical engineering from Oklahoma State University, USA. Currently, $\mathrm{He}$ is an Assistant Professor at Kasetsart University, Bang Kean Campus, Thailand. His research interests are electrical machines dynamics, power system dynamic and power system satiability. 